\title{
Caracterização florístico-estrutural e síndromes de dispersão de espécies lenhosas de remanescente de Cerradão
}

\author{
Weuler Alves VASCONCELOS ${ }^{1 *}$, Sabrina do Couto de MIRANDA ${ }^{2}$, \\ Carlos de Melo SILVA-NETO ${ }^{3}$, Priscila Bezerra de SOUZA ${ }^{1}$
}

\author{
${ }^{1}$ Universidade Federal de Tocantins, Gurupi, TO, Brasil. (Orcid: *; 0000-0003-4602-3139) \\ 2Universidade Estadual de Goiás, Palmeiras de Goiás, GO, Brasil. (Orcid: 0000-0002-3861-6674) \\ ${ }^{3}$ Instituto Federal de Educação, Ciência e Tecnologia, Cidade de Goiás, GO, Brasil. (Orcid: 0000-0001-8624-3836) \\ *E-mail: weulervasconcelos@gmail.com (Orcid: 0000-0002-1602-1194)
}

Recebido em 24/03/2020; Aceito em 15/06/2020; Publicado em 31/07/2020.

\begin{abstract}
RESUMO: Entender a composição florístico-estrutural e as síndromes de dispersão de um remanescente fornecem subsídios para futuros planos de recuperação e conservação. Desta forma, objetivou-se analisar a composição florística, a estrutura da vegetação lenhosa e as síndromes de dispersão de um remanescente de cerradão localizado em Palmeiras de Goiás, GO. Para tal, foram alocadas 10 parcelas de $20 \times 50 \mathrm{~m}$ onde todos indivíduos com diâmetro da base $(\mathrm{Db}) \geq 5 \mathrm{~cm}$ ingressaram na amostragem, sendo medido ainda seu diâmetro à altura do peito (DAP) e altura. Foram amostrados 2.070 indivíduos distribuídos em 109 espécies, 86 gêneros e 49 famílias, sendo Fabaceae a família mais abundante. As espécies Tapirira guianensis e Xylopia aromatica se destacaram em Valor de Importância na área. A distribuição diamétrica apresentou-se no formato de "J"invertido e a distribuição de altura com tendência normal, mostrando uma comunidade auto regenerativa. A síndrome de dispersão predominante foi a zoocoria, com 55,5\% das espécies. Os resultados encontrados corroboram a importância das áreas de reserva legal e a necessidade de sua manutenção como estratégia de conservação/preservação ambiental.
\end{abstract}

Palavras-chave: fitofisionomia florestal; zoocoria; anemocoria; comunidade.

\section{Floristic-structural characterization and dispersion syndromes of woody species of Cerradão remanescent}

\begin{abstract}
Understanding the floristic-structural composition and the dispersion syndromes of a remnant provides subsidies for future recovery and conservation plans. Thus, the aim was to analyze the floristic composition, woody vegetation structure and dispersion syndromes of a cerradão remnant located in Palmeiras de Goiás, GO. For this, 10 plots of $20 \times 50 \mathrm{~m}$ were allocated where all individuals with base diameter (Db) $\geq$ $5 \mathrm{~cm}$ entered the sampling, and their diameter at breast height (DAP) and height were also measured. We sampled 2,070 individuals distributed in 109 species, 86 genera and 49 families, Fabaceae being the most abundant family. The species Tapirira guianensis and Xylopia aromatica stood out in Valor de Importância in the area. The diametric distribution was presented as "J"-inverted and the height distribution with normal tendency, showing a self-regenerative community. The predominant dispersion syndrome was zoocoria, with $55.5 \%$ of the species. The results found corroborate the importance of the legal reserve areas and the need for their maintenance as a conservation/environmental preservation strategy.
\end{abstract}

Keywords: forest phytophysiognomy; zoochory; anemocory; assembly.

\section{INTRODUÇÃO}

Diante do contexto nacional e internacional, o Cerrado tem ganhado destaque devido a sua grande extensão geográfica no Brasil, diversidade associada e serviços ambientais ofertados. Dentre as savanas mundiais, apresenta a flora mais rica com cerca de 12 mil espécies de plantas vasculares (MENDONÇA et al., 2008). A riqueza de espécies está relacionada ao alto grau de endemismos e heterogeneidade espacial expressa por um mosaico de fitofisionomias que englobam desde formações florestais (mata ciliar, mata de galeria, mata seca e cerradão), savânicas (cerrado sentido restrito, parque de cerrado, palmeiral e vereda) até campestres (campo limpo, campo sujo e campo rupestre) (RIBEIRO; WALTER, 2008).
Atualmente todas as fitofisionomias do bioma apresentam-se ameaçadas, principalmente pela conversão da vegetação nativa em outros usos antrópicos, como pastagens e agricultura. Contudo, é importante destacar que o cerradão é a fitofisionomia mais fragmentada e com maior índice de degradação, visto que ocorre principalmente em áreas de terreno plano, com solos em sua maioria de boa fertilidade e de fácil mecanização (SANO et al., 2010). Apesar das características de formação florestal, cobertura de dossel entre $50 \%$ e $90 \%$, e árvores que comumente atingem entre 8 e $15 \mathrm{~m}$, floristicamente o cerradão apresenta maior similaridade com o cerrado sentido restrito (RIBEIRO; WALTER, 2008).

Martins (2009) afirma que o passo inicial para a preservação e recuperação de áreas naturais é o 
monitoramento através do levantamento fitossociológico, no qual, os dados obtidos por meio da composição florísticoestrutural da comunidade fornecem subsídios para futuros planos de recuperação e conservação (GIMENEZ; GODOY, 2007). Complementando, Zavala et al. (2017) afirmam que conhecer a biodiversidade e a estrutura de áreas naturais se faz urgente para propor medidas de conservação.

Além disso, compreender a diversificação de frutos e as diferentes estratégias de dispersão de diásporos é importante no processo de recuperação e manutenção natural de áreas nativas (HOWE; SMALLWOOD, 1982; FLEMING, 1991; JORDANO et al., 2006). Os modos de dispersão associados aos estratos de vegetação e os tipos de ambientes influenciam diretamente na capacidade dispersiva e na distribuição geográfica das espécies vegetais (FENNER, 1985; LORTS et al., 2008), influenciando assim no sucesso futuro da manutenção de áreas recuperadas.

Diante do contexto apresentado, este trabalho objetivou analisar a composição florística, a estrutura vertical e horizontal, e as síndromes de dispersão de diásporos das espécies lenhosas em um remanescente de cerradão localizado na região core do bioma Cerrado, visando colaborar para a realização de projetos futuros com vistas à recuperação de áreas degradadas.

\section{MATERIAL E MÉTODOS}

\section{1. Área de estudo}

O estudo foi realizado em um remanescente de cerradão localizado na Fazenda Buritis, munícipio de Palmeiras de Goiás, sob as coordenadas $16^{\circ} 51^{\prime} 23.89^{\prime \prime S}$ e 4959'29.95"O. Trata-se de uma área particular inserida em uma região predominantemente agrícola, designada como reserva legal, com área de aproximadamente 52 hectares e altitude média de $571 \mathrm{~m}$ (Figura 1). O clima da região é do tipo Aw (tropical úmido, com estação chuvosa no verão e seca no inverno) segundo a classificação de Köppen-Geiger, com precipitação média anual igual a $1.457 \mathrm{~mm}$ e a temperatura média de 25,6 ${ }^{\circ} \mathrm{C}$ (CLIMADATA.ORG, 2018).

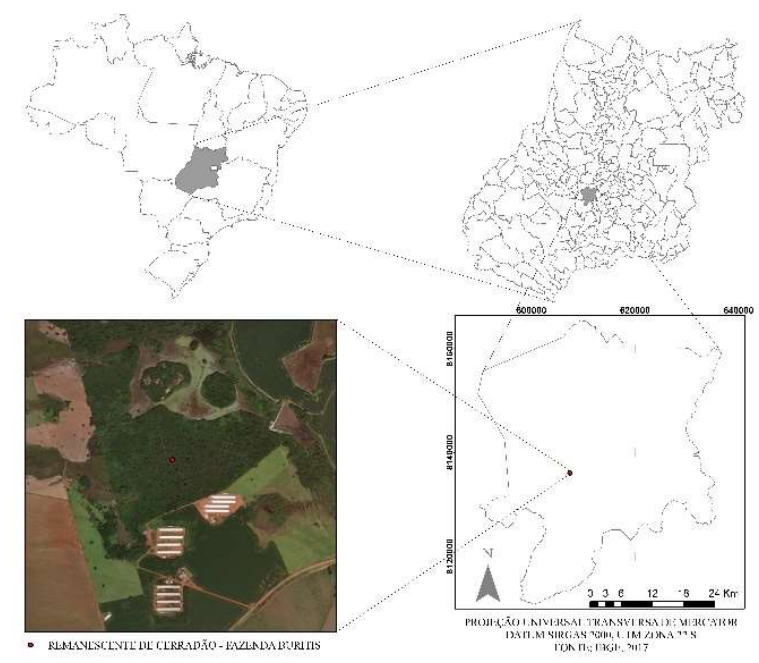

Figura 1. Mapa de Localização do remanescente de cerradão estudado no município de Palmeiras de Goiás, Goiás, Brasil. Figure 1. Location map of the remnant of cerradão studied in the municipality of Palmeiras de Goiás, Goiás, Brazil.

\subsection{Coleta e análise de dados}

Foram instaladas dez parcelas de 20 x $50 \mathrm{~m}$ cada, totalizando um esforço amostral igual a um hectare, onde todos os indivíduos lenhosos com diâmetro da base (Db), medidos a $30 \mathrm{~cm}$ do solo, igual ou superior a $5 \mathrm{~cm}$ foram mensurados, desconsiderando lianas e palmeiras, e incluindo os indivíduos mortos em pé (FELFILI et al., 2005). As parcelas foram instaladas aleatoriamente ao longo da área tendo como referência uma estrada central já existente e mantendo a distância mínima de $50 \mathrm{~m}$ das bordas, seguindo recomendações do Manual de Parcelas Permanentes dos Biomas Cerrado e Pantanal (FELFILI et al., 2005). No local o proprietário possui um pequeno apiário para produção de mel, ocasionalmente há entrada de gado e retirada de madeira.

Assim como realizado por Miranda et al. (2017), os indivíduos ingressantes no critério de inclusão tiveram também o diâmetro à altura do peito (DAP) e altura mensurados. O diâmetro foi medido com auxílio de suta de alumínio e a altura com régua retrátil, ou ainda com auxílio de hipsômetro digital. A altura foi considerada como a projeção vertical da base do tronco até o ramo mais alto ou folhagem.

Os parâmetros fitossociológicos densidade, frequência e dominância, absolutas e relativas, e o valor de importância (VI) foram calculados de acordo com Müeller-Dombois; Ellenberg (1974), com auxílio do programa Microsoft Excel.

Para as análises de distribuição de altura e diâmetro da comunidade foram adotados os intervalos fixos de $5 \mathrm{~cm}$ para o diâmetro e um metro para as classes de altura, visto a facilidade para comparações com outros dados disponíveis na literatura (FELFILI; REZENDE, 2003; MIRANDA et al., 2013; MIRANDA et al., 2017).

A identificação dos indivíduos foi feita in loco, quando possível, ou pela análise de material botânico coletado por meio de comparações com coleções disponíveis no Herbário da Universidade de Brasília (UB) ou ainda consultas a especialistas. $O$ material testemunho foi depositado no herbário didático da Universidade Estadual de Goiás, Unidade Universitária de Palmeiras de Goiás. As espécies foram classificadas em famílias de acordo com o sistema do Angiosperm Phylogeny Group IV (APG IV, 2016) e os nomes de todas as espécies foram atualizadas por meio de consultas a "Lista de Espécies da Flora do Brasil" (REFLORA, 2020) e ao "Tropicos" (https://www.tropicos.org/).

A fim de verificar a abrangência da amostragem florística foi elaborada a curva de acumulação de espécies (KENT; COKER, 1992). Para uma melhor demonstração dos esforços foram geradas três curvas: uma de acumulação de espécies (curva do coletor) e duas de estimativas de riqueza através dos índices de Jackenife 1 e Bootstrap resampling, descritos em Magurran; McGill (2011), sendo estas estimadas com auxílio do programa R 3.6.2 (R CORE TEAM, 2019).

Para caracterizar a diversidade florística foram analisadas a riqueza $(\mathrm{S})$, a diversidade alfa através do Índice de Shannon $\left(\mathrm{H}^{\prime}\right)$ e a Uniformidade de Pielou (J') (MAGURRAN; MCGILL, 2011), sendo calculados com o auxílio do programa MVSP.

As similaridades florística e estrutural entre as parcelas amostradas foram calculadas através dos índices de Sørensen (MAGURRAN, 2004) e Czekanowski (KENT; COKER, 1992) que se baseiam, respectivamente, na presença de 
espécies e valores de densidade, também calculados no programa MVSP.

As espécies lenhosas amostradas na área foram classificadas quanto às síndromes de dispersão primária levando-se em consideração o tipo de diásporo e suas adaptações. Adotando os critérios morfológicos definidos por Pijl (1982) foram categorizadas em: 1) zoocóricas espécies que possuem características relacionadas à dispersão por animais; 2) anemocóricas - apresentam mecanismos que facilitam a dispersão pelo vento; e 3) autocóricas - espécies que dispersam os diásporos por gravidade ou apresentam mecanismos de auto dispersão, como a abertura explosiva. Para a classificação analisou-se morfologicamente os diásporos coletados em campo, bem como, informações disponíveis na literatura especializada (PINHEIRO; RIBEIRO, 2001; KUHLMANN, 2016).

\section{RESULTADOS}

A curva de acumulação pelos estimadores Jacknife e Bootstrap indicou que $89 \%$ e $80 \%$ das espécies da área, respectivamente, foram amostradas (Figura 2). A densidade na área foi de 2.070 ind.ha ${ }^{-1}$, sendo 1.721 indivíduos lenhosos vivos e 349 mortos em pé (Tabela 1). Os indivíduos vivos estão distribuídos em 109 espécies, 86 gêneros e 49 famílias (Tabela 1). As parcelas apresentaram alta similaridade em termos de composição florística (Figura 3-A), contudo em termos estruturais as parcelas foram separadas em dois grupos com similaridade abaixo de $50 \%$, o grupo constituído pelas parcelas CD7, CD9 e CD10 distinguiu-se das demais. A similaridade entre estes conjuntos foi de $32 \%$, ressaltando diferenças em termos de densidade (Figura $3-\mathrm{B}$ ).

A diversidade florística calculada pelo índice de Shannon \& Wiener $\left(\mathrm{H}^{\prime}\right)$ foi de 3,85 nats.ind $^{-1}$ e a uniformidade de Pielou (J') 0,82. As famílias com maior abundância em número de espécies foram Fabaceae, Anacardiaceae, Annonaceae, Malpighiaceae e Vochysiaceae, que juntas somaram 54,27\% dos indivíduos vivos amostrados. Na área de estudo a densidade total foi de 2.070 ind.ha $^{-1}$ e a dominância total de $16,70 \mathrm{~m}^{2} \cdot \mathrm{ha}^{-1}$ (Tabela 1). Os indivíduos mortos em pé ocuparam a primeira colocação no valor de importância (VI), 10,17\% do total, e apresentaram uma densidade de 349 ind.ha ${ }^{-1}$ (16,9\% do total).

A área de estudo apresenta uma alta riqueza (109 espécies), em detrimento disso, sete espécies (6,4\% do total) foram responsáveis por $28,3 \%$ do VI total (Tabela 1). São consideradas com alto valor de importância na área as espécies Tapirira guianensis Aubl., Xylopia aromatica (Lam.) Mart., Curatella americana L., Sclerolobium paniculatum Vogel, Copaifera langsdorffii Desf., Byrsonima pachyphylla A. Juss. e Roupala montana Aubl. (Tabela 1). Juntas estas espécies somaram $34,7 \%$ da densidade e $37,8 \%$ da dominância da área. Dentre as espécies amostradas, 20 (18,3\% do total) apresentaram-se representadas na área por apenas um indivíduo (Tabela 1).

Observou-se, com base nas síndromes de dispersão primária dos diásporos, o predomínio de espécies zoocóricas (55,5\%), seguidas de anemocóricas $(36,1 \%)$ e autocóricas $(1,9 \%)$. As espécies sem informação ou identificadas apenas em nível de família somaram 6,5\% do total e não impactaram os dados analisados (Tabela 1 ).

O diâmetro de todos os indivíduos arbóreos amostrados variou de $5,0 \mathrm{~cm}$ a $55,5 \mathrm{~cm}$, com média de $10,52 \mathrm{~cm}$ e coeficiente de variação (CV) de 50,9\%. A distribuição diamétrica possui uma distribuição exponencial negativa, conhecida como "J" invertido (Figura 4). A altura dos indivíduos lenhosos amostrados variou de 0,65 a $15 \mathrm{~m}$, com média de $5,85 \mathrm{~m}$ e $\mathrm{CV}$ de $41,2 \%$. A maioria dos indivíduos $(58 \%)$ concentrou-se nas classes de altura entre 4,0 e $8,0 \mathrm{~m}$ (Figura 5).

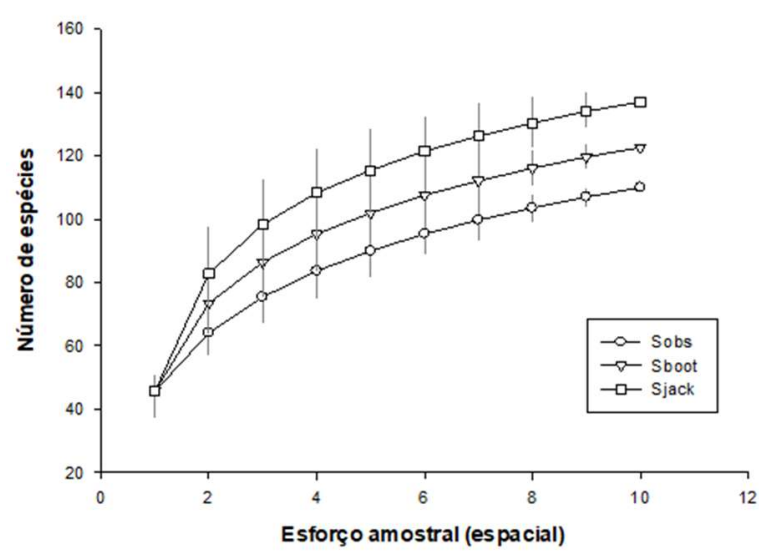

Figura 2. Curvas de acumulação (Sobs) e estimativas de riqueza (Sjack e Sboot) para a vegetação lenhosa amostrada em remanescente de cerradão no município de Palmeiras de Goiás-GO. Figure 2. Accumulation curves (Sobs) and richness estimates (Sjack and Sboot) for the woody vegetation sampled in remnants of cerradão in the municipality of Palmeiras de Goiás-GO.

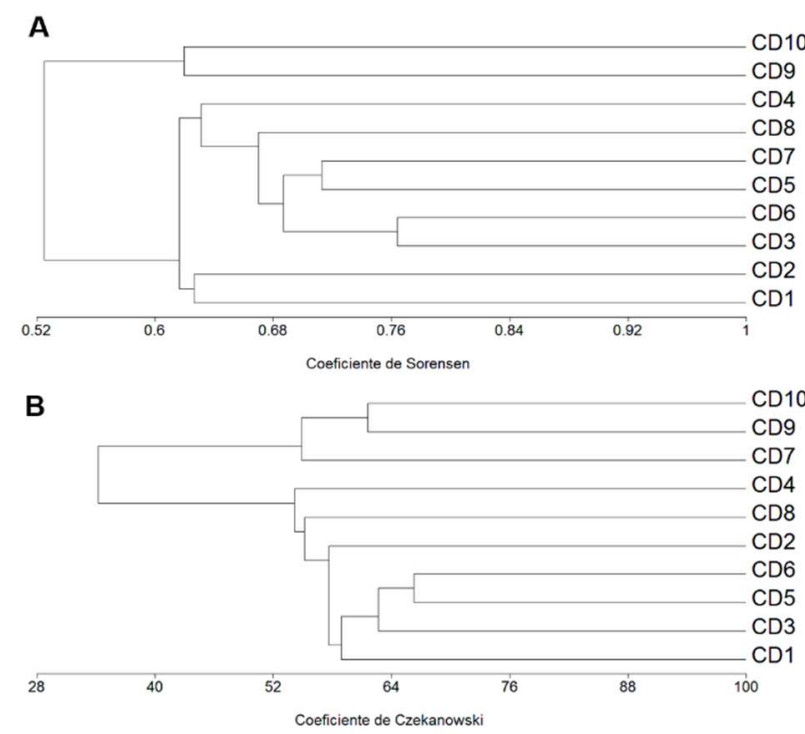

Figura 3. Dendrogramas de classificação da vegetação lenhosa de um remanescente de cerradão no município de Palmeiras de GoiásGO produzidos a partir dos índices de Sørensen (A) e Czekanowski (B).

Figure 3. Dendrograms for the classification of woody vegetation of a remnant of cerradão in the municipality of Palmeiras de GoiásGO produced from the Sørensen (A) and Czekanowski (B) indexes. 
Tabela 1. Parâmetros fitossociológicos e síndrome de dispersão dos indivíduos arbóreos amostrados em remanescente de cerradão em Palmeiras de Goiás. Em que: DA = densidade absoluta; DR = densidade relativa (\%); DoA = dominância absoluta; DoR $=$ dominância relativa; $\mathrm{FA}=$ frequência absoluta $(\%) ; \mathrm{FR}=$ Frequência relativa $(\%) ; \mathrm{VI}=$ Valor de importância; $\mathrm{ANE}=$ Anemocoria; $\mathrm{AUTO}=$ Autocoria; $\mathrm{ZOO}=$ Zoocoria; $\mathrm{SI}=$ sem informação.

Table 1. Phytosociological parameters and dispersion syndrome of tree individuals sampled in remnants of cerradão in Palmeiras de Goiás. Where: $\mathrm{DA}=$ absolute density; $\mathrm{DR}=$ relative density $(\%) ; \mathrm{DoA}=$ absolute dominance; $\mathrm{DoR}=$ relative dominance; FA $=$ absolute frequency $(\%) ; \mathrm{FR}=$ Relative frequency $(\%) ; \mathrm{IVI}=$ Importance value; $\mathrm{ANE}=$ Anemocoria; $\mathrm{AUTO}=$ Autocoria; $\mathrm{ZOO}=\mathrm{Zoocoria} ; \mathrm{SI}=$ no information.

\begin{tabular}{|c|c|c|c|c|c|c|c|c|}
\hline Espécies & DA & DR & FA & FR & DoA & DoR & VI & Síndromes \\
\hline Morta & 349 & 16,9 & 100 & 2,2 & 1,92 & 11,5 & 30,5 & - \\
\hline Tapirira guianensis Aubl. & 162 & 7,83 & 100 & 2,2 & 1,48 & 8,84 & 18,9 & $\mathrm{ZOO}$ \\
\hline Xylopia aromatica (Lam.) Mart. & 119 & 5,75 & 100 & 2,2 & 0,91 & 5,42 & 13,4 & $\mathrm{ZOO}$ \\
\hline Curatella americana L. & 83 & 4,01 & 100 & 2,2 & 0,85 & 5,04 & 11,3 & $\mathrm{ZOO}$ \\
\hline Sclerolobium paniculatum Vogel* & 66 & 3,19 & 90 & 1,98 & 0,99 & 5,91 & 11,1 & ANE \\
\hline Copaifera langsdorffii Desf. & 92 & 4,44 & 70 & 1,54 & 0,81 & 4,85 & 10,8 & $\mathrm{ZOO}$ \\
\hline Byrsonima pachyphylla A. Juss. & 109 & 5,27 & 80 & 1,76 & 0,63 & 3,74 & 10,8 & $\mathrm{ZOO}$ \\
\hline Roupala montana Aubl. & 88 & 4,25 & 90 & 1,98 & 0,67 & 3,97 & 10,2 & ANE \\
\hline Annona crassiflora Mart. & 40 & 1,93 & 100 & 2,2 & 0,62 & 3,72 & 7,86 & $\mathrm{ZOO}$ \\
\hline Qualea grandiflora Mart. & 30 & 1,45 & 100 & 2,2 & 0,53 & 3,19 & 6,84 & ANE \\
\hline Bowdichia virgililoides Kunth & 29 & 1,4 & 90 & 1,98 & 0,53 & 3,13 & 6,52 & ANE \\
\hline Byrsonima coccolobifolia Kunth & 109 & 5,27 & 80 & 1,76 & 0,63 & 3,74 & 10,8 & $\mathrm{ZOO}$ \\
\hline Alibertia edulis var. edulis (Rich.) A.Rich. & 52 & 2,51 & 100 & 2,2 & 0,13 & 0,79 & 5,5 & $\mathrm{ZOO}$ \\
\hline Eriotheca gracilipes (K. Schum.) A. Robyns & 14 & 0,68 & 70 & 1,54 & 0,52 & 3,11 & 5,32 & ANE \\
\hline Terminalia argentea Mart. & 43 & 2,08 & 80 & 1,76 & 0,24 & 1,41 & 5,25 & ANE \\
\hline Guapira noxia (Netto) Lundell & 29 & 1,4 & 90 & 1,98 & 0,3 & 1,81 & 5,19 & $\mathrm{ZOO}$ \\
\hline Astronium fraxinifolium Schott & 34 & 1,64 & 100 & 2,2 & 0,18 & 1,1 & 4,94 & ANE \\
\hline Ouratea glaucescens Engl. & 34 & 1,64 & 70 & 1,54 & 0,26 & 1,56 & 4,74 & $\mathrm{ZOO}$ \\
\hline Emmotum nitens (Benth.) Miers & 21 & 1,01 & 40 & 0,88 & 0,42 & 2,5 & 4,39 & $\mathrm{ZOO}$ \\
\hline Dimorphandra mollis Benth. & 19 & 0,92 & 70 & 1,54 & 0,29 & 1,74 & 4,2 & $\mathrm{ZOO}$ \\
\hline Qualea multiflora Mart. & 30 & 1,45 & 90 & 1,98 & 0,09 & 0,53 & 3,96 & ANE \\
\hline Myrsine guianensis (Aubl.) Kuntze & 23 & 1,11 & 80 & 1,76 & 0,18 & 1,06 & 3,93 & $\mathrm{ZOO}$ \\
\hline Platypodium elegans Vogel & 30 & 1,45 & 70 & 1,54 & 0,14 & 0,85 & 3,84 & ANE \\
\hline Micropholis venulosa (Mart. \& Eichler ex Miq.) Pierre & 25 & 1,21 & 80 & 1,76 & 0,13 & 0,8 & 3,77 & $\mathrm{ZOO}$ \\
\hline Magonia pubescens A. St.-Hil. & 15 & 0,72 & 70 & 1,54 & 0,24 & 1,44 & 3,7 & ANE \\
\hline Pseudobombax tomentosum (Mart.) Robyns & 16 & 0,77 & 80 & 1,76 & 0,17 & 1,01 & 3,54 & ANE \\
\hline Platbymenia reticulata Benth. & 17 & 0,82 & 80 & 1,76 & 0,15 & 0,87 & 3,45 & ANE \\
\hline Qualea parviflora Mart. & 18 & 0,87 & 60 & 1,32 & 0,19 & 1,16 & 3,35 & ANE \\
\hline Cordiera macrophylla (K. Schum.) Kuntze & 24 & 1,16 & 80 & 1,76 & 0,05 & 0,3 & 3,22 & $\mathrm{ZOO}$ \\
\hline Diospyros hispida A. DC. & 18 & 0,87 & 50 & 1,1 & 0,2 & 1,18 & 3,15 & $\mathrm{ZOO}$ \\
\hline Machaerium acutifolium Vogel & 18 & 0,87 & 50 & 1,1 & 0,18 & 1,05 & 3,02 & ANE \\
\hline Styrax ferrugineus Nees \& Mart. & 23 & 1,11 & 60 & 1,32 & 0,07 & 0,41 & 2,84 & $\mathrm{ZOO}$ \\
\hline Tabebuia aurea (Silva Manso) Benth. \& Hook.f. ex S.Moore & 17 & 0,82 & 60 & 1,32 & 0,1 & 0,59 & 2,73 & ANE \\
\hline Scutia of buxifolia Reissek & 12 & 0,58 & 80 & 1,76 & 0,04 & 0,26 & 2,6 & ANE \\
\hline Diospyros burchellii Hiern & 12 & 0,58 & 50 & 1,1 & 0,13 & 0,77 & 2,45 & $\mathrm{ZOO}$ \\
\hline Acosmium dasycarpum (Vogel) Yakovlev & 15 & 0,72 & 50 & 1,1 & 0,09 & 0,53 & 2,36 & ANE \\
\hline Connarus suberosus Planch. & 14 & 0,68 & 60 & 1,32 & 0,05 & 0,3 & 2,29 & $\mathrm{ZOO}$ \\
\hline Handroantbus ochraceus (Cham.) Mattos & 13 & 0,63 & 50 & 1,1 & 0,09 & 0,56 & 2,29 & ANE \\
\hline Machaerium opacum Vogel & 18 & 0,87 & 50 & 1,1 & 0,18 & 1,05 & 3,02 & ANE \\
\hline Erythroxylum daphnites Mart. & 15 & 0,72 & 60 & 1,32 & 0,03 & 0,19 & 2,24 & $\mathrm{ZOO}$ \\
\hline Guapira graciliflora (Mart. Ex J.A. Schmidt) Lundell & 15 & 0,72 & 50 & 1,1 & 0,05 & 0,32 & 2,14 & $\mathrm{ZOO}$ \\
\hline Vatairea macrocarpa (Benth.) Ducke & 9 & 0,43 & 50 & 1,1 & 0,07 & 0,44 & 1,97 & ANE \\
\hline Sapindaceae 1 & 9 & 0,43 & 40 & 0,88 & 0,1 & 0,62 & 1,93 & SI \\
\hline Eriotheca pubescens (Mart. \& Zucc.) Schott \& Endl. & 5 & 0,24 & 30 & 0,66 & 0,13 & 0,78 & 1,68 & ANE \\
\hline Tocoyena formosa (Cham. \& Schltdl.) K.Schum. & 9 & 0,43 & 50 & 1,1 & 0,02 & 0,14 & 1,68 & $\mathrm{ZOO}$ \\
\hline Pseudobombax longiflorum (Mart.) A. Robyns & 7 & 0,34 & 50 & 1,1 & 0,04 & 0,21 & 1,65 & ANE \\
\hline Luebea divaricata Mart. & 8 & 0,39 & 40 & 0,88 & 0,06 & 0,38 & 1,65 & ANE \\
\hline Lithraea molleoides (Vell.) Engl. & 7 & 0,34 & 50 & 1,1 & 0,02 & 0,12 & 1,56 & $\mathrm{ZOO}$ \\
\hline Miconia albicans (Sw.) Steud. & 6 & 0,29 & 50 & 1,1 & 0,02 & 0,1 & 1,49 & $\mathrm{ZOO}$ \\
\hline Coussarea bydrangeifolia (Benth.) Müll. Arg. & 9 & 0,43 & 40 & 0,88 & 0,02 & 0,15 & 1,46 & $\mathrm{ZOO}$ \\
\hline Myracrodruon urundewva Allemão & 8 & 0,39 & 30 & 0,66 & 0,06 & 0,36 & 1,41 & ANE \\
\hline Couepia grandiflora (Mart. \& Zucc.) Benth. ex Hook. f. & 5 & 0,24 & 40 & 0,88 & 0,03 & 0,2 & 1,32 & $\mathrm{ZOO}$ \\
\hline Pterodon pubescens (Benth.) Benth. & 6 & 0,29 & 30 & 0,66 & 0,06 & 0,34 & 1,29 & ANE \\
\hline Ouratea bexasperma (A. St.-Hil.) Baill. & 4 & 0,19 & 40 & 0,88 & 0,03 & 0,18 & 1,26 & $\mathrm{ZOO}$ \\
\hline Aspidosperma tomentosum Mart. & 6 & 0,29 & 40 & 0,88 & 0,01 & 0,08 & 1,25 & ANE \\
\hline Rubiaceae 1 & 9 & 0,43 & 30 & 0,66 & 0,02 & 0,14 & 1,24 & SI \\
\hline Alchornea glandulosa Poepp. & 7 & 0,34 & 30 & 0,66 & 0,04 & 0,21 & 1,21 & $\mathrm{ZOO}$ \\
\hline Stryphnodendron adstringens (Mart.) Coville & 6 & 0,29 & 20 & 0,44 & 0,08 & 0,47 & 1,2 & $\mathrm{ZOO}$ \\
\hline Siparuna guianensis Aubl. & 4 & 0,19 & 40 & 0,88 & 0,01 & 0,07 & 1,15 & $\mathrm{ZOO}$ \\
\hline Kielmeyera coriacea Mart. \& Zucc. & 5 & 0,24 & 30 & 0,66 & 0,04 & 0,24 & 1,14 & ANE \\
\hline Byrsonima sericea DC. & 3 & 0,14 & 30 & 0,66 & 0,05 & 0,32 & 1,12 & $\mathrm{ZOO}$ \\
\hline
\end{tabular}




\begin{tabular}{|c|c|c|c|c|c|c|c|c|}
\hline Espécies & $\mathrm{DA}$ & DR & FA & FR & DoA & DoR & VI & Síndromes \\
\hline Hymenaea stigonocarpa Mart. ex Hayne & 3 & 0,14 & 30 & 0,66 & 0,05 & 0,31 & 1,12 & $\mathrm{ZOO}$ \\
\hline Tabebuia impetiginosa (Mart. ex DC.) Standl. & 5 & 0,24 & 20 & 0,44 & 0,07 & 0,4 & 1,08 & ANE \\
\hline Protium beptaphyllum (Aubl.) Marchand & 3 & 0,14 & 30 & 0,66 & 0,04 & 0,22 & 1,03 & $\mathrm{ZOO}$ \\
\hline Callisthene fasciculata Mart & 4 & 0,19 & 10 & 0,22 & 0,09 & 0,57 & 0,98 & ANE \\
\hline Cardiopetalum calopbyllum Schltdl. & 5 & 0,24 & 30 & 0,66 & 0,01 & 0,06 & 0,97 & $\mathrm{ZOO}$ \\
\hline Erythroxylum suberosum A. St.-Hil. & 5 & 0,24 & 20 & 0,44 & 0,02 & 0,15 & 0,83 & $\mathrm{ZOO}$ \\
\hline Terminalia glabrescens Mart. & 4 & 0,19 & 20 & 0,44 & 0,03 & 0,17 & 0,81 & ANE \\
\hline Cecropia sp. 1 & 3 & 0,14 & 20 & 0,44 & 0,03 & 0,16 & 0,75 & $\mathrm{ZOO}$ \\
\hline Licania apetala (E.Mey.) Fritsch & 3 & 0,14 & 20 & 0,44 & 0,01 & 0,07 & 0,65 & ZOO \\
\hline Vochysia tucanorum Mart. & 3 & 0,14 & 20 & 0,44 & 0,01 & 0,07 & 0,65 & ANE \\
\hline Rhamnidium elaeocarpum Reissek & 3 & 0,14 & 20 & 0,44 & 0,01 & 0,06 & 0,65 & $\mathrm{ZOO}$ \\
\hline Eugenia sp.1 & 3 & 0,14 & 20 & 0,44 & 0,01 & 0,06 & 0,65 & $\mathrm{ZOO}$ \\
\hline Neea theifera Oerst. & 2 & 0,1 & 20 & 0,44 & 0,02 & 0,1 & 0,64 & $\mathrm{ZOO}$ \\
\hline Virola sebifera Aubl. & 3 & 0,14 & 20 & 0,44 & 0,001 & 0,03 & 0,61 & $\mathrm{ZOO}$ \\
\hline Myrsine gardneriana A. DC. & 2 & 0,1 & 20 & 0,44 & 0,01 & 0,06 & 0,6 & $\mathrm{ZOO}$ \\
\hline Physocalymma scaberrimum Pohl & 2 & 0,1 & 20 & 0,44 & 0,01 & 0,05 & 0,58 & ANE \\
\hline Aegiphila lhotzkiana Cham. & 2 & 0,1 & 20 & 0,44 & 0,01 & 0,04 & 0,58 & $\mathrm{ZOO}$ \\
\hline Fabaceae 1 & 2 & 0,1 & 20 & 0,44 & 0,001 & 0,03 & 0,56 & SI \\
\hline Cybistax antisyphilitica (Mart.) Mart. & 2 & 0,1 & 20 & 0,44 & 0,001 & 0,02 & 0,56 & ANE \\
\hline Sapindaceae 2 & 2 & 0,1 & 20 & 0,44 & 0,001 & 0,02 & 0,56 & SI \\
\hline Eugenia aurata O.Berg & 2 & 0,1 & 20 & 0,44 & 0,001 & 0,01 & 0,55 & $\mathrm{ZOO}$ \\
\hline Plenckia populnea Reissek & 1 & 0,05 & 10 & 0,22 & 0,05 & 0,27 & 0,54 & ANE \\
\hline Stryphnodendron rotundifolium Mart. & 3 & 0,14 & 10 & 0,22 & 0,02 & 0,14 & 0,51 & $\mathrm{ZOO}$ \\
\hline Baubinia sp.1 & 4 & 0,19 & 10 & 0,22 & 0,01 & 0,06 & 0,47 & AUTO \\
\hline Rourea induta Planch. & 2 & 0,1 & 10 & 0,22 & 0,02 & 0,14 & 0,45 & ZOO \\
\hline Trichilia sp.1 & 1 & 0,05 & 10 & 0,22 & 0,03 & 0,18 & 0,45 & $\mathrm{ZOO}$ \\
\hline Myrtaceae & 3 & 0,14 & 10 & 0,22 & 0,01 & 0,08 & 0,44 & $\mathrm{ZOO}$ \\
\hline Malpighiaceae 1 & 1 & 0,05 & 10 & 0,22 & 0,02 & 0,13 & 0,4 & SI \\
\hline Pouteria ramiflora (Mart.) Radlk. & 1 & 0,05 & 10 & 0,22 & 0,02 & 0,12 & 0,39 & $\mathrm{ZOO}$ \\
\hline Byrsonima basiloba A. Juss. & 1 & 0,05 & 10 & 0,22 & 0,02 & 0,11 & 0,38 & ZOO \\
\hline Chrysopbyllum marginatum (Hook. \& Arn.) Radlk. & 2 & 0,1 & 10 & 0,22 & 0,01 & 0,04 & 0,36 & $\mathrm{ZOO}$ \\
\hline Heteropterys byrsonimifolia A. Juss. & 2 & 0,1 & 10 & 0,22 & 0,01 & 0,04 & 0,35 & ANE \\
\hline Schefflera sp.1 & 1 & 0,05 & 10 & 0,22 & 0,01 & 0,05 & 0,32 & $\mathrm{ZOO}$ \\
\hline Aspidosperma cuspa (Kunth) S.F.Blake & 1 & 0,05 & 10 & 0,22 & 0,01 & 0,04 & 0,3 & ANE \\
\hline Tabebuia chrysotricha (Mart. ex DC.) Standl. & 1 & 0,05 & 10 & 0,22 & 0,01 & 0,03 & 0,3 & ANE \\
\hline Davilla elliptica A.St.-Hil. & 1 & 0,05 & 10 & 0,22 & 0,01 & 0,03 & 0,3 & $\mathrm{ZOO}$ \\
\hline Rubiaceae 2 & 1 & 0,05 & 10 & 0,22 & 0,001 & 0,02 & 0,28 & SI \\
\hline Maytenus floribunda Reissek & 1 & 0,05 & 10 & 0,22 & 0,001 & 0,01 & 0,28 & ZOO \\
\hline Baubinia sp.2 & 4 & 0,19 & 10 & 0,22 & 0,01 & 0,06 & 0,47 & AUTO \\
\hline Ouratea castaneifolia (DC.) Engl. & 1 & 0,05 & 10 & 0,22 & 0,001 & 0,01 & 0,28 & ZOO \\
\hline Sapotaceae & 1 & 0,05 & 10 & 0,22 & 0,001 & 0,01 & 0,28 & SI \\
\hline Byrsonima verbascifolia (L.) DC. & 1 & 0,05 & 10 & 0,22 & 0,001 & 0,01 & 0,28 & $\mathrm{ZOO}$ \\
\hline Myrcia rostrata DC. & 1 & 0,05 & 10 & 0,22 & 0,001 & 0,01 & 0,28 & $\mathrm{ZOO}$ \\
\hline Lafoensia pacari A. St.-Hil. & 1 & 0,05 & 10 & 0,22 & 0,001 & 0,01 & 0,28 & ANE \\
\hline Enterolobium gummiferum (Mart.) J.F.Macbr. & 1 & 0,05 & 10 & 0,22 & 0,001 & 0,01 & 0,28 & $\mathrm{ZOO}$ \\
\hline Matayba guianensis Aubl. & 1 & 0,05 & 10 & 0,22 & 0,001 & 0,01 & 0,28 & $\mathrm{ZOO}$ \\
\hline Ilex ferruginea Hand.-Mazz. & 1 & 0,05 & 10 & 0,22 & 0,001 & 0,01 & 0,28 & $\mathrm{ZOO}$ \\
\hline Aspidosperma sp.1 & 1 & 0,05 & 10 & 0,22 & 0,001 & 0,01 & 0,28 & ANE \\
\hline TOTAL & 2.070 & 100 & 4.540 & 100 & 16,70 & 100 & 300 & - \\
\hline
\end{tabular}

* Tachigali subvelutina (Benth.) Oliveira-Filho - sinônima aceita.

\section{DISCUSSÃO}

A análise a partir dos estimadores de riqueza ressaltou que o método de amostragem empregado possibilitou o levantamento de cerca de $80 \%$ da riqueza florística da área. Mesmo com os valores relativamente altos dos estimadores de riqueza, não foi verificada a completa estabilização das curvas (Figura 1), tal característica é comum em ambientes com alta diversidade de espécies e de grupos taxonômicos (famílias e gêneros). Oliveira; Batalha (2005), Borges; Shepherd (2005) e Silva et al. (2008) afirmam que a nãoassintotização das curvas é um reflexo da alta diversidade alfa e baixa frequência absoluta de espécies.

A densidade de indivíduos lenhosos vivos obtida neste estudo ficou dentro da faixa de variação encontrada para outras áreas de cerradão amostradas com a mesma metodologia, entre 1.458 ind.ha ${ }^{-1}$ e 2.424 ind.ha ${ }^{-1}$ conforme compilação apresentada por Miranda et al. (2017).

As árvores mortas em pé desempenham papel importante na manutenção de áreas naturais, pois servem de abrigo para a fauna e contribuem para a manutenção dos estoques de carbono (VASCONCELOS et al., 2019). A alta densidade de indivíduos mortos em pé amostrados na área pode estar relacionada com a dinâmica natural das populações (ciclo de vida das espécies arbóreas) que compõem esta comunidade, bem como, com fatores estocásticos. É importante ressaltar que em campo não se verificou sinais evidentes de distúrbios frequentes, como o fogo, ou presença de gado nas parcelas. 


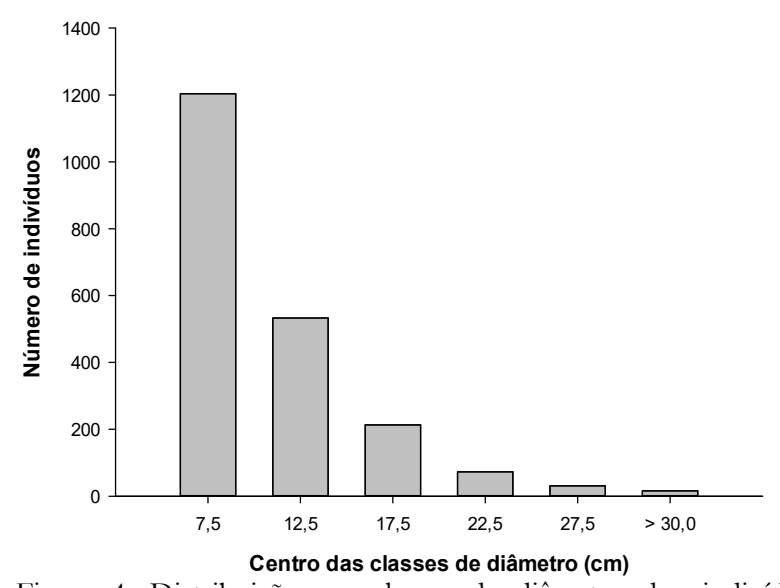

Figura 4. Distribuição em classes de diâmetro dos indivíduos lenhosos amostrados em remanescente de cerradão em Palmeiras de Goiás, Goiás.

Figure 4. Distribution in diameter classes of woody individuals sampled in remnants of cerradão in Palmeiras de Goiás, Goiás.

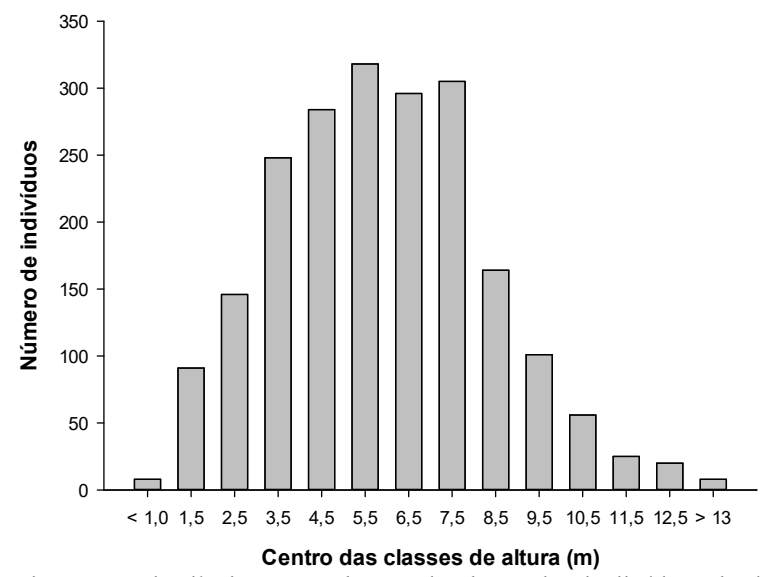

Figura 5. Distribuição em classes de altura dos indivíduos lenhosos amostrados em remanescente de cerradão em Palmeiras de Goiás, Goiás.

Figure 5. Distribution in height classes of woody individuals sampled in remnants of cerradão in Palmeiras de Goiás, Goiás.

As análises de similaridade revelaram que as parcelas amostradas no cerradão possuem alta semelhança na composição florística, contudo das dez parcelas, três (parcelas 7, 9 e 10) apresentaram-se distintas das demais em termos estruturais (Figura 3). Nestas três parcelas as espécies C. largsdorffi, Emmotum nitens (Benth.) Miers, Platypodium elegans Vogel e T. guianensis apresentaram os maiores valores de densidade, respectivamente, $89,1 \%, 95,2 \%, 80 \%$ e $63,6 \%$ do total amostrado para cada espécie. Das espécies amostradas no cerradão, nove ocorreram apenas nestas parcelas, a saber: Aspidosperma cuspa (Kunth) S.F. Blake, Callisthene fasciculata Mart, Eugenia aurata O.Berg, Matayba guianensis Aubl., Maytenus floribunda Reissek, Myrcia rostrata DC., Ouratea castaneifolia (DC.) Engl., Plenckia populnea Reissek e Rhamnidium elaeocarpum Reissek. Além disso, 31 espécies não foram amostradas nestas parcelas, com destaque para Ouratea glaucescens Engl. que ocorreu em todas as parcelas à exceção das três diferenciadas. Também ressaltamos que foram identificadas quatro espécies de Ipê (Handroanthus ocbraceus (Cham.) Mattos, Tabebuia aurea (Silva Manso) Benth. \&
Hook.f. ex S.Moore, Tabebuia chrysotricha (Mart. ex DC.) Standl. e Tabebuia impetiginosa (Mart. ex DC.) Standl.), porém nenhuma delas ocorreu nas três parcelas mencionadas, corroborando a diferenciação. Assim, são necessários outros estudos buscando entender atributos locais, por exemplo associados ao substrato, que podem ter colaborado para a diferenciação discutida.

O índice de diversidade obtido ficou próximo ao limite superior da faixa de variação para outras áreas. Miranda et al. (2017) comparando 12 áreas de cerradão no Brasil observou variação nos valores entre 2,13 e 4,00 nats.ind ${ }^{-1}$. Além disso, pode-se destacar o estudo de Giácomo et al. (2015) na Estação Ecológica de Pirapitinga, MG e de Vasconcelos et al. (2019) na Bacia Hidrográfica do Rio Vermelho, GO, que obtiveram valores de 3,22 nats.ind $^{-1}$ e 4,00 nats.ind ${ }^{-1}$, respectivamente.

As variações nos valores de diversidade encontrados pelos diferentes estudos podem ser explicadas pelo fato do cerradão compartilhar espécies com outras fitofisionomias do bioma Cerrado. A matriz florística onde os remanescentes estão inseridos influencia significativamente a composição de espécies, além disso fatores abióticos tais como clima, relevo e tipo de solo também contribuem para as diferentes encontradas.

Fabaceae compõe o grupo de famílias com maior variedade de espécies e maior frequência no presente estudo, bem como, em outros levantamentos realizados no Cerrado (SOUZA et al., 2010; VASCONCELOS et al., 2019). Espécies dessa família possuem alta capacidade de nodulação e associação com bactérias fixadoras de nitrogênio, com isso apresentam maior aclimatação em locais com baixo teor de macronutriente, principalmente em áreas do Cerrado, por apresentar solo, na maioria das vezes, pobre em nutrientes (CORDEIRO, 2000).

O fragmento de vegetação estudado na região de Palmeiras de Goiás, sudoeste do estado de Goiás é caracterizado como Cerradão. Os parâmetros estruturais da vegetação e a composição de espécies reforçam sua definição como este tipo de vegetação, segundo critérios definidos por Ribeiro; Walter (2008), e Ferreira et al. (2017). De acordo com Ferreira et al. (2017), as espécies Qualea grandiflora Mart., C. langsdorffii, E. nitens, Qualea parviflora Mart., Cordiera macrophylla (K. Schum.) Kuntze, Tachigali subvelutina (Benth.) Oliveira-Filho (sinônima S. paniculatum), Simarouba versicolor A. St.-Hil., P. elegans, Bowdichia virgililoides Kunth, Qualea multiflora Mart., X. aromatica, Machaerium acutifolium Vogel, M. rostrata, Astronium fraxinifolium Schott e Aspidosperma tomentosum Mart. são consideradas indicadoras de cerradão. À exceção de $S$. versicolor, todas as espécies indicadoras foram encontradas no cerradão estudado em Palmeiras de Goiás, corroborando a identidade florística da fitofisionomia.

Os resultados encontrados por Solórzano et al. (2012), Giácomo et al. (2015), Miguel et al. (2017) e Vasconcelos et al. (2019) em áreas de cerradão mostraram que as principais espécies são E. nitens, Q. grandiflora, Tachigali vulgaris L.F. Gomes da Silva \& H.C. Lima, C. langsdorffii, Vatairea macrocarpa (Benth.) Ducke e B. virgilioides. Estas espécies em conjunto podem ser utilizadas para caracterizar o cerradão (SÓLORZANO et al., 2012), assim como Caryocar brasiliense (RIBEIRO; WALTER, 2008), o que corrobora a composição florística obtida no presente estudo.

Com relação às espécies localmente raras, do total de espécies amostradas, 20 (18,5\% do total) apresentaram 
densidade igual a 1 ind.ha ${ }^{-1}$ e totalizam $2,24 \%$ do IVI total. Entretanto nenhuma destas espécies consta no Livro Vermelho da Flora do Brasil - Plantas Raras do Brasil (MARTINELLI et al., 2014).

A zoocoria foi a síndrome de dispersão mais comum dentre as espécies lenhosas amostradas, corroborando o fato de se tratar de um ambiente florestal geralmente mais atrativo para a fauna, o que assegura o sucesso de colonização de novas áreas. Em seguida observou-se para a área amostrada a anemocoria, isso decorrente de o cerradão não apresentar dossel totalmente fechado, permitindo a entrada de luz e circulação de vento, o que possibilita a dispersão por anemocoria. Oliveira et al. (2018) em estudo em área de Cerrado no sul do estado do Tocantins encontraram resultados próximos ao presente trabalho, sendo a zoocoria a síndrome de maior frequência entre as espécies amostradas.

Observa-se predominância da zoocoria em ambientes florestais, o que reflete a associação da vegetação com a fauna, sendo fonte de alimento para os animais, além de abrigo e local para nidificação/reprodução, possibilitando assim a manutenção da diversidade faunística local. Desta forma, necessita-se de estudos que relacionem a florística, a estrutura e as síndromes de dispersão das espécies para compreender a magnitude dos serviços ambientais oferecidos pelos remanescentes naturais e propor medidas de conservação e de recuperação de áreas próximas. Os dados gerados no presente trabalho ganham ainda mais importância no sentido que se trata de um remanescente de vegetação nativa inserido em uma zona predominantemente agrícola e com raros estudos.

A distribuição diamétrica dos indivíduos amostrados apresentou característica exponencial negativa, conhecida como "J" invertido, esse padrão indica que o recrutamento é maior que a mortalidade e que a comunidade se apresenta auto regenerativa (Figura 4) (ASSUNÇÃO; FELFILI, 2004), dados esses semelhantes aos encontrados no cerradão de Itapirapuã-GO (VASCONCELOS et al., 2019). Esse padrão mostra uma comunidade que permite se reestruturar de forma natural, sendo capaz de suportar pressões externas. Os valores encontrados para altura dos indivíduos lenhosos (Figura 5) corroboram que se trata de uma formação florestal, onde a altura média ficou acima dos 5,5 metros.

\section{CONCLUSÕES}

O remanescente de cerradão estudado em Palmeiras de Goiás mostrou-se com alta densidade, riqueza e diversidade de espécies lenhosas. Corroborando a literatura, Fabaceae foi a família mais abundante na área. A maior densidade foi representada pelos indivíduos mortos em pé que possuem o maior VI (Valor de Importância), seguido das espécies Tapirira guianensis e Xylopia aromatica, sendo estas espécies importantes em termos de recuperação de áreas degradadas, pois são dispersas por aves e mamíferos. A distribuição diamétrica apresentou-se no formato de "J" invertido e a distribuição de altura com tendência normal, mostrando uma comunidade auto regenerativa.

As análises das síndromes de dispersão de diásporos das espécies lenhosas revelaram predominância da zoocoria (55,5\%), tal aspecto contribui para a construção de matrizes de conservação e fontes para a recuperação de áreas degradadas, justificando estudos sobre florística, estrutura e síndrome de dispersão em remanescentes. Os resultados encontrados também corroboram a importância das áreas de reserva legal, bem como, a necessidade de sua manutenção como estratégia de conservação/preservação ambiental.

\section{AGRADECIMENTOS}

$O$ primeiro autor agradece à Coordenação de Aperfeiçoamento de Pessoal de Nível Superior (CAPES) pela concessão da bolsa de estudo e ao proprietário da Fazenda Buriti por permitir o estudo na área.

\section{REFERÊNCIAS}

APG IV_THE ANGIOSPERM PHYLOGENY GROUP. An update of the Angiosperm Phylogeny Group classification for the orders and families of flowering plants: APG IV. Botanical Journal of the Linnean Society, London, v. 181, n. 1, p. 1-20, 2016. DOI: https://dx.doi.org/10.1111/boj.12385

ASSUNÇÃO S. L.; FELFILI J. M. Fitossociologia de um fragmento de Cerrado sensu stricto na APA do Paranoá, DF, Brasil. Acta Botanica Brasilica, São Paulo, v. 18, n. 4, p. 903-909, 2004. DOI: https://dx.doi.org/10.1590/S0102-33062004000400021

BORGES, H. B. N.; SHEPHERD, G. J. Flora e estrutura do estrato lenhoso numa comunidade de Cerrado em Santo Antônio do Leverger, MT, Brasil. Brazilian Journal of Botany, São Paulo, v. 28, n. 1, p. 61-74, 2005. DOI: https://doi.org/10.1590/S0100-84042005000100006

CLIMA-DATA.ORG. Clima: Palmeiras de Goiás. Disponível em: <https://pt.climatedata.org/location/43436/>. Acesso em: 30 jul. 2018.

CORDEIRO, L. Fixação de nitrogênio em leguminosas ocorrentes no Cerrado. In: KLEI, A. L. Eugem Warming e o Cerrado brasileiro: um século depois. São Paulo: Editora USP, p. 131-145. 2000.

FELFILI, J. M.; CARVALHO, F. A.; HAIDAR, R. F. Manual para o monitoramento de parcelas permanentes nos biomas Cerrado e Pantanal. Universidade de Brasília, Departamento de Engenharia Florestal. Brasília: 2005. 51 p.

FELFILI, J. M.; REZENDE, R. P. Conceitos e métodos em fitossociologia. Universidade de Brasília, Departamento de Engenharia Florestal, 2003. 68 p.

FENNER, M. Seed ecology. New York, Chapman and Hall. 1985. $151 \mathrm{p}$.

FERREIRA, R. Q. S.; CAMARGO, M. O.; TEIXEIRA, P. R.; SOUZA, P. B. de; SOUZA, D. J. de. Diversidade florística do estrato arbustivo arbóreo de três áreas de cerrado sensu stricto, Tocantins. Desafios, Palmas, v. 4, n. 2, p. 69-82, 2017. DOI: http:/ /dx.doi.org/10.20873/uft.23593652.2017v4n2p69

FLEMING, T. H. Fruiting plant-frugivore mutualism: the evolutionary theater and the ecological play. In: PRICE, P. W.; et al. (Eds.) Plant-animal interactions: evolutionary ecology in tropical and temperate regions. NewYork, J. Wiley and Sons, 1991. p. 119-144.

GIÁCOMO, R. G.; PEREIRA, M. G.; CARVALHO, D. C.; MEDEIROS, V. S.; GAUI, T. D. Florística e Fitossociologia em Áreas de cerradão e Mata Mesofítica na Estação Ecológica de Pirapitinga, MG. Floresta e Ambiente, Seropédica, v. 22, n. 3, p.287-298, 2015. DOI: https://dx.doi.org/10.1590/2179-8087.067913

GIMENEZ, V. M. M.; GODOY, S. A. P. de. Diversidade da Vegetação Regenerante de um Cerrado após Plantio de 
Exóticas em Luiz Antônio (SP). Revista Brasileira de Biociências, Porto Alegre, v. 5, n. 2, p. 729-731, 2007.

HOWE, H. F.; SMALLWOOD, J. Ecology of seeds dispersal. Annual Review of Ecology and Systematics, Palo Alto, v. 13, p. 201-228, 1982. DOI: https://dx.doi.org/10.1146/annurev.es.13.110182.0012 21

JORDANO, P.; GALETTI, M.; PIZO, M. A.; SILVA, W. R. Ligando frugivoria e dispersão de sementes à biologia da conservação. In: ROCHA, C. D. F.; BERGALLO, H. D.; VAN SLUYS, M.; ALVES, M. A. S. (Ed.). Biologia da Conservação: Essências. São Carlos: Rima Editora, 2006, p. 411-436.

KENT, M.; COKER, P. Vegetation description and analysis: a practical approach. 2. ed. Oxford: John Wiley \& Sons, 1992. 428 p.

KUHLMANN, M. Estratégias de dispersão de sementes no Bioma Cerrado: Considerações Ecológicas e Filogenéticas. 2016. 353f. Tese (Doutorado) Programa de Pós-Graduação em Botânica, Instituto de Ciências Biológicas, Universidade de Brasília, Brasília, 2016.

LORTS, C. M.; BRIGGEMAN, T.; SANG, T. Evolution of fruit types and seed dispersal: a phylogenetic and ecological snapshot. Journal of Systematics and Evolution, Beijing, v. 46, n. 3, p. 396-404, 2008.

MAGURRAN, A. E. Measuring biological diversity. Oxford: Blackwell Publishing. Oxford: Blackwell Science, 2004. 256 p.

MAGURRAN, A. E.; MCGILL, B. J. Biological Diversity: frontiers in measurement and assessment. Oxford: Oxford University Press, 2011. 345 p.

MARTINELLI, G.; MESSINA, T.; SANTOS FILHO, L. Livro vermelho da flora do Brasil - Plantas raras do Cerrado. Rio de Janeiro: Instituto de Pesquisas Jardim Botânico do Rio de Janeiro. CNCFlora, 2014. 320 p.

MARTINS, S. V. Recuperação de área degradadas: ações em Áreas de Preservação Permanente, voçorocas, taludes rodoviários e de mineração. Viçosa, MG: Aprenda Fácil, 2009. 27 p.

MENDONÇA, R. C.; FELFILI, J. M.; WALTER, B. M.; SILVA-JÚNIOR, M. C. S.; REZENDE, A. V.; FILGUEIRAS, T. S.; NOGUEIRA, P. E.; FAGG, C. W. Flora Vascular do Cerrado. In: SANO, S. M.; ALMEIDA, S. P. (Eds.). Cerrado: ambiente e flora Planaltina, DF: EMBRAPA-CPAC, 2008. p. 289-556.

MIGUEL, E. P.; REZENDE, A. V.; LEAL, F. A.; MATRICARDI, E. A. T.; ENSINAS, J. M. I.; MIRANDA, J. F. N. Floristic, structural, and allometric equations to estimate arboreal volume and biomass in a cerradão site. Semina: Ciências Agrárias, Londrina, v. 38, n. 4, p. 1691-1702, 2017. DOI: http:/ /dx.doi.org/10.5433/1679-0359.2017v38n4p1691

MIRANDA, S. C.; SILVA JÚNIOR, M. C.; DECARVALHO, P. S. O efeito da proteção do fogo na estrutura da vegetação lenhosa de uma área de cerrado sentido restrito no Brasil Central. Heringeriana, Brasília, v. 7, n. 1, p. 61-72, 2013.

MIRANDA, S. C.; DE-CARVALHO, P. S.; SILVA JÚNIOR, M. C. Atributos ecológicos de espécies amplamente distribuídas em cerrado sentido restrito: Capítulo I. In: RIBON, A. A.; DE-CARVALHO, P. S.;
MIRANDA, S. C. Tópicos em Conservação e Manejo do Cerrado. Goiânia: Ed. Kelps, 2017, p. 13-37.

MÜLLER-DOMBOIS, D.; ELLENBERG, H. Aims and methods of vegetation ecology. New York: J. Wiley \& Sons, 1974. 574 p.

OLIVEIRA, F. F.; BATALHA, M. A. Lognormal abundance distribution of woody species in a cerrado fragment (São Carlos, SW Brazil). Brazilian Journal of Botany, São Paulo, v. 28, n. 1, p. 39-45, 2005. DOI: https://dx.doi.org/10.1590/S0100-84042005000100004

OLIVEIRA, L. M.; SOUSA, R. M.; CORREA, N. E. R.; SANTOS, A. F.; GIONGO, M. Florística e síndromes de dispersão de um fragmento de Cerrado ao sul do estado do Tocantins. Revista Scientia Agraria Paranaensis, Marechal Cândido Rondon, v. 17, n. 1, p. 104-111, 2018.

PIJL, V. D. L. Principles of dispersal in higher plants. 3 ed. New York: Springer Verlag, 1982. 228 p.

PINHEIRO, F.; RIBEIRO, J. F. Síndromes de dispersão de sementes em Matas de Galeria do Distrito Federal. In: RIBEIRO, J. F.; FONSECA, C. E. L.; SOUSA-SILVA, J. C. Cerrado: caracterização e recuperação de Matas de Galeria. Planaltina: Embrapa Cerrados. 2001. p. 335375.

$\mathrm{R}$ Core Team. R: A language and environment for statistical computing. R Foundation for Statistical Computing, Vienna, Austria, 2019. Disponível em: https://www.R-project.org/. 2019. Acesso em: 10 jul. 2019.

REFLORA. Lista de espécies da flora do Brasil. Jardim Botânico do Rio de Janeiro. Disponível em: http://floradobrasil.jbrj.gov.br/2012/. Acesso em: 11 março. 2019.

RIBEIRO, J. F.; WALTER, B. M. T. As principais fitofisionomias do bioma Cerrado. In: SANO, S. M.; ALMEIDA S. P.; RIBEIRO, J. F. (Ed.). Cerrado: ecologia e flora. Embrapa Cerrados, Brasília, 2008. p. 153-212.

SANO, E. E.; ROSA, R.; BRITO, J. L. S.; FERREIRA, L. G. Land cover mapping of the tropical savanna region in Brazil. Environmental Monitoring and Assessment, Amstedam, v. 166, p. 113-124, 2010. DOI: http://dx.doi.org/10.1007/s10661-009-0988-4

SILVA, H. G.; FIGUEIREDO, N.; ANDRADE, G. V. A. Estrutura da vegetação de um cerradão e a heterogeneidade regional do Cerrado no Maranhão, Brasil. Revista Árvore, Viçosa, v. 32, n. 5, 2008. DOI: https://dx.doi.org/10.1590/S0100-67622008000500017 SOLÓRZANO, A.; PINTO, J. R. R.; FELFILI, J. M.; HAY, J. D. V. Perfil florístico e Estrutural do componente lenhoso em seis áreas de cerradão ao longo do bioma Cerrado. Acta Botânica Brasílica, Feira de Santana, v. 26, n. 2, p. 328-341, 2012. DOI: https://dx.doi.org/10.1590/S0102-33062012000200009

SOUZA, P. B.; SAPORETTI JUNIOR, A. W.; SOARES, M. P.; VIANA, R. H. O.; CAMARGOS, V. L.; MEIRA NETO, J. A. A. Florística de uma área de cerradão na floresta nacional de Paraopeba - Minas Gerais. Cerne, Lavras, v. 16, n. 1, p. 86-93, 2010. DOI: https://dx.doi.org/10.1590/S0104-77602010000100010

VASCONCELOS, W. A. et al. A vegetação lenhosa de cerradão na bacia hidrográfica do Rio Vermelho, Goiás: Capítulo I. In: MIRANDA, S. C.; DE-CARVALHO, P. S.; RIBON, A. A. Tópicos em conservação e manejo 
do cerrado: biodiversidade, solos e uso sustentável.

Goiânia: Editora Kelps, 2019. p. 9-30.

ZAVALA, C. B. R.; FERNANTES, S. S. L.; PEREIRA, Z.

V.; SILVA, S. M. Análise fitogeográfica da flora arbustivo-arbórea em ecótono no Planalto da Bodoquena, MS, Brasil. Ciência Florestal, Santa Maria, v. 27, n. 3, p. 907-921, 2017. DOI: https://dx.doi.org/10.5902/1980509828640 